\title{
Correlation Inequalities for Ising Ferromagnets with Symmetries *
}

\author{
Gerhard C. Hegerfeldt \\ Institut für Theoretische Physik, Universität Göttingen, D-3400 Göttingen \\ Federal Republic of Germany
}

\begin{abstract}
We derive new correlation inequalities for even Ising ferromagnets whose interaction is invariant under some symmetry transformation and satisfies a growth condition. The recent results of Schrader [1] and Messager and Miracle-Sol [2] for the nearest neighbour (n.n.) Ising model reappear as a special case. In addition we obtain monotonicity of $\left\langle\sigma_{0} \sigma_{j}\right\rangle$ under translation of $j$ perpendicular to diagonal hyperplanes and the inequality $\left\langle\sigma_{0} \sigma_{j}\right\rangle \geqq\left\langle\sigma_{0} \sigma_{\left(\Sigma\left|j_{v}\right|, \underline{0}\right)}\right\rangle$ for n.n. and other interactions.
\end{abstract}

\section{Introduction}

Recently, Schrader [1] and, independently, Messager and Miracle-Sol [2] found some new interesting inequalities for the n.n. Ising model. Let $\vartheta$ denote reflection,

$$
\vartheta \sigma_{j}=\sigma_{\left(-j_{1}, \underline{j}\right)},
$$

and let $f_{v}(\sigma)$ be a polynomial in $\left\{\sigma_{j} ; j_{1} \geqq 0\right\}, v=1, \ldots$, with nonnegative coefficients. Then, for the n.n. Ising model [1]

$$
\left\langle\prod_{v}\left(f_{v} \pm \vartheta f_{v}\right)\right\rangle \geqq 0
$$

for any combination of \pm signs. In particular $[1,2],\left\langle\sigma_{0} \sigma_{\left(j_{1}, j\right)}\right\rangle$ is monotone decreasing in $j_{1}>0$.

In this paper we analyze general even Ising ferromagnets which are invariant under some symmetry transformation, e.g., a reflection. Under a certain growth condition on the interaction we derive new correlation inequalities which contain those of $[1,2]$ as special cases (Theorems 3.1 and 3.2). The monotonicity properties of correlations which we obtain are of interest in themselves. We hope that the other inequalities may prove helpful to study the effects of boundary conditions as in [2] for the n.n. Ising model.

Let $\mathbb{Z}^{d}$ denote the $d$-dimensional square lattice with unit spacing. For $i \in \mathbb{Z}^{d}, \sigma_{i}$ denotes the "spin at site $i$ " with probability distribution given by some measure $v_{i}$ on

* Dedicated to Professor G. Ludwig's 60th birthday 
$\mathbb{R}$. The interaction between the spins in a finite volume $\Lambda$ is taken as

$$
H_{\Lambda}=\sum_{i, k \in \Lambda} J_{i k} \sigma_{i} \sigma_{k}-\sum_{i \in \Lambda} \mu_{i} \sigma_{i}
$$

and the corresponding correlation functions are defined as

$$
\left\langle\sigma_{i^{1}} \ldots \sigma_{i^{n}}\right\rangle_{\Lambda}=Z_{\Lambda}^{-1} \int \prod_{i \in \Lambda} d v_{i}\left(\sigma_{i}\right) e^{-\beta H} \sigma_{i^{1}} \ldots \sigma_{i^{n}}
$$

where $Z_{A}$ is a normalization constant such that $\langle 1\rangle_{A}=1$. As it stands, Equation (1.2) refers to free boundary conditions. By making $\Lambda$ a torus one deals with periodic boundary conditions.

We speak of a (general) Ising ferromagnet with constant external field $\mu$ if

$$
J_{i k} \leqq 0 \quad \text { for } \quad i \neq k, \quad \mu_{i} \equiv \mu,
$$

and of an even Ising ferromagnet if in addition the single spin distributions are even and all equal,

$$
d v_{i}(x) \equiv d v(x)=d v(-x) .
$$

The term "the n.n. Ising model" refers to an even Ising ferromagnet with

$$
J_{i j}=\left\{\begin{array}{cl}
-J<0 & \text { for }|i-j|=1 \\
0 & \text { otherwise }
\end{array}\right.
$$

and a next nearest neighbour (n.n.n.) interaction is of the form

$$
J_{i j}= \begin{cases}J_{i j} & \text { for } 0<|i-j| \leqq 2 \\ 0 & \text { otherwise }\end{cases}
$$

In Section 2 we derive a fairly general "Main Lemma" involving arbitrary symmetry transformations. It is shown that the usual Griffith's (or GKS) inequalities are obtained from it as a special case. In Section 3 the Main Lemma is applied to even Ising ferromagnets, in particular to those with reflection invariant interaction (Theorems 3.1 and 3.2).

\section{The Main Lemma}

The derivation of the Main Lemma is based on the following Lemma 2.3 which is quite well-known ${ }^{1}$. We include a proof for completeness only.

Lemma 2.1. Let $v$ be an even positive measure on $\mathbb{R}$ such that every polynomial is integrable. Then, for any integers $n, m \geqq 0$

$$
\int\left(x+x^{\prime}\right)^{n}\left(x-x^{\prime}\right)^{m} d v(x) d v\left(x^{\prime}\right) \geqq 0 .
$$

Proof. By $x^{\prime} \rightarrow-x^{\prime}$ symmetry the integral is symmetric in $n, m$. For $m$ odd it vanishes by $\left(x, x^{\prime}\right) \rightarrow\left(x^{\prime}, x\right)$ symmetry. If $n, m$ are even the integrand is non-negative. QED.

Lemma 2.2. Let $I$ be a finite index set and $a_{i}, b_{i} \in \mathbb{C}, i \in I$. Then

$$
\prod a_{i( \pm)} \prod b_{i}=2^{-|I|+1} \sum_{\substack{A \subset I \\|A| \text { even (odd) }}} \prod_{i \in A}\left(a_{i}-b_{i}\right) \prod_{j \in I \backslash A}\left(a_{j}+b_{j}\right) \text {. }
$$

Proof. Express $a_{i}, b_{i}$ by $a_{i} \pm b_{i}$ and expand the 1.h.s.

Cf. Ginibre [3] or Simon [4], p. $275 \mathrm{ff}$. 
Lemma 2.3. Let $v_{0 \alpha}, v_{i}, \alpha=1, \ldots, N_{0}, i=1, \ldots, N$ be even positive measures such that every polynomial is integrable. Let $f_{k}\left(x_{0}, x\right), k=1, \ldots, M$, be polynomials in $\left\{x_{0 \alpha}\right\},\left\{x_{i}\right\}$ with positive coefficients. Then

$$
\int \prod d v_{0 \alpha}\left(x_{0 \alpha}\right) \prod d v_{i}\left(x_{i}\right) \prod d v_{i}\left(x_{i}^{\prime}\right) \prod\left(f_{k}\left(x_{0}, x\right) \pm f_{k}\left(x_{0}, x^{\prime}\right)\right) \geqq 0
$$

for any combination of \pm signs.

Proof. Obviously it suffices to consider monomials. The $x_{0}$-factors can be written in front of the product sign, and integration over $x_{0}$ gives a non-negative factor. Applying Lemma 2.2 to the remaining integrand and then using Lemma 2.1 shows that also the remaining integral is non-negative. QED.

Main Lemma. Let $\Lambda$ be a finite set, and let $\Phi$ be a bijection of $\Lambda$. Let $\Lambda_{0} \subset \Lambda$ be the set of invariant elements and let $\Lambda=\Lambda_{0} \cup \Lambda_{+} \cup \Lambda_{-}$be some disjoint decomposition with

$$
\Phi \Lambda_{+}=\Lambda_{-} .
$$

Let $J=\left\{J_{i j} ; i, j \in \Lambda\right\}$ be a real symmetric matrix satisfying

(i) $J_{\Phi i \Phi j}=J_{i j}$ for all $i, j \in \Lambda$ (invariance)

(ii) $J_{i j} \leqq 0$ for $i \neq j$ (ferromagnet)

(iii) $\left|J_{i j}\right| \geqq\left|J_{i \Phi j}\right|$ for $i \neq j, i, j \in \Lambda_{+}$(growth condition)

(iv) $J_{i \Phi j}=J_{j \Phi i}$ for $i, j \in \Lambda_{+}{ }^{2}$.

Let the normalized measure $\mu_{\Lambda}$ on $\mathbb{R}^{|\Lambda|}$ be given by

$$
d \mu_{\Lambda}(x)=Z_{\Lambda}^{-1} e^{-\sum J_{i j} x_{i} x_{j}+\sum \mu_{\imath} x_{i}} \prod_{i \in \Lambda} d v_{i}\left(x_{i}\right)
$$

where $Z_{A}$ is a normalization constant, where

$$
\mu_{i} \geqq 0^{3}, \quad \mu_{\Phi i}= \pm \mu_{i}, \quad i \in \Lambda_{+} \cup \Lambda_{0},
$$

and where $\left\{v_{i}, i \in \Lambda\right\}$ are even positive measures on $\mathbb{R}$ with

$$
v_{\Phi i}=v_{i}
$$

and such that every polynomial in $\left\{x_{i}, i \in \Lambda\right\}$ is integrable with respect to $\mu_{\Lambda}$. For any function $f$ on $\mathbb{R}^{|\Lambda|}$ let

$$
(\phi f)\left(\left\{x_{i}\right\}\right)=f\left(\left\{x_{\Phi i}\right\}\right) \text {. }
$$
Then

Now let $f_{1}, \ldots, f_{n}$ be any polynomials in $\left\{x_{i} ; i \in \Lambda_{+} \cup \Lambda_{0}\right\}$ with positive coefficients.

$$
\left\langle\prod_{k}\left(f_{k} \pm \phi f_{k}\right)\right\rangle_{\Lambda} \equiv \int d \mu_{\Lambda} \prod_{k}\left(f_{k} \pm \phi f_{k}\right) \geqq 0
$$

for any combination of \pm signs.

Proof. First we assume that all $v_{i}$ 's have compact support. For $\alpha \in \Lambda_{0}$, we put $y_{0 \alpha}=x_{\alpha}$ and, for $i \in \Lambda_{+}, y_{i}=x_{i}$ and $y_{i}^{\prime}=x_{\Phi i}$. Then

$$
\left(f_{k} \pm \phi f_{k}\right)(x)=f_{k}\left(y_{0}, y\right) \pm f_{k}\left(y_{0}, y^{\prime}\right) \text {. }
$$

If $\Phi^{2}=$ identity, (iv) is automatically fulfilled

One can equally well suppose $\mu_{i} \leqq 0$, by $x_{i} \rightarrow-x_{i}$ symmetry 
We decompose $\sum J_{i j} x_{i} x_{j}$ into a sum of terms where $i, j$ run through $\Lambda_{0}, \Lambda_{+}$, and $\Lambda_{-}$ and then introduce the new variables. As an example we consider the term

$$
\begin{aligned}
2 \sum_{i \in \Lambda_{+}, k \in \Lambda_{-}} J_{i k} x_{i} x_{k} & =2 \sum_{i, j \in \Lambda_{+}} J_{i \Phi j} x_{i} x_{\Phi j} \\
& =2 \sum_{i, j \in \Lambda_{+}} J_{i \Phi j} y_{i} y_{j} .
\end{aligned}
$$

By Condition (iv), the r.h.s. can be written as

$$
\sum_{i, j \in \Lambda_{+}} J_{i \Phi j}\left(y_{i}+y_{i}^{\prime}\right)\left(y_{j}+y_{j}^{\prime}\right)-\sum_{i, j \in \Lambda_{+}} J_{i \Phi j}\left(y_{i} y_{j}+y_{i}^{\prime} y_{j}\right) \text {. }
$$

In the other terms we extract the diagonal contributions. Invariance of $J$ and $v_{i}$ under $\Phi$ finally yields

$$
\begin{aligned}
& d \mu_{\Lambda}= Z_{\Lambda}^{-1} \exp \left\{-\sum_{\alpha \neq \beta \in \Lambda_{0}} J_{\alpha \beta} y_{0 \alpha} y_{0 \beta}-2 \sum_{\alpha \in \Lambda_{0}, i \in \Lambda_{1}} J_{\alpha i} y_{0 \alpha}\left(y_{i}+y_{i}^{\prime}\right)\right. \\
&-\sum_{i, j \in \Lambda_{+}} J_{i \Phi_{j}}\left(y_{i}+y_{i}^{\prime}\right)\left(y_{i}+y_{j}^{\prime}\right)-\sum_{i \neq j \in \Lambda_{+}}\left(J_{i j}-J_{i \Phi j}\right)\left(y_{i} y_{j}+y_{i}^{\prime} y_{j}^{\prime}\right) \\
&\left.+\sum_{\alpha \in \Lambda_{0}} \mu_{\alpha} y_{0 \alpha}+\sum_{i \in \Lambda_{+}} \mu_{i}\left(y_{i} \pm y_{j}^{\prime}\right)\right\} \\
& \prod_{\alpha \in \Lambda_{0}} e^{-J_{\alpha \alpha} y_{0 \alpha}^{2}} d v_{\alpha}\left(y_{0 \alpha}\right) \prod_{i \in \Lambda_{+}} e^{-\left(J_{i i}-J_{i \Phi_{i}}\right) y_{i}} d v_{i}\left(y_{i}\right) \\
& \prod_{i \in \Lambda_{+}} e^{-\left(J_{i i}-J_{i \Phi}\right) y_{i}^{\prime} 2} d v_{i}\left(y_{i}^{\prime}\right) .
\end{aligned}
$$

The expressions under the products signs define new even measures. Hence, expanding the remaining exponentials in Equation (2.7), the 1.h.s. of Equation (2.5) becomes a sum of terms to each of which Lemma 2.3 can be applied by (iii) and since

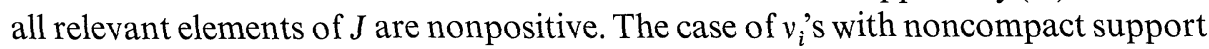
is now obtained by approximation and limits. QED.

Corollary 2.1. Equation (2.5) of the Main Lemma still holds if the $f_{k}$ 's are elements of the positive cone generated by functions $h\left(x_{i}\right), i \in \Lambda_{+} \cup \Lambda_{0}$, of the form

(i) $h$ is odd and monotone increasing on $\mathbb{R}$ or

(ii) $h$ is positive, even and monotone increasing on the positive half-axis.

Proof. Lemma 2.3 still holds for such functions $f_{k}{ }^{4}$ so the proof literally carries over to this case.

Corollary 2.2. Let $f_{1}, f_{2}$ be functions as in the Main Lemma or as in Corollary 2.1. Then

$$
\left\langle f_{1} f_{2}\right\rangle_{A}+\left\langle\phi f_{1} \phi f_{2}\right\rangle_{\Lambda} \geqq\left|\left\langle f_{1} \phi f_{2}\right\rangle_{A}+\left\langle f_{2} \phi f_{1}\right\rangle_{A}\right| \text {. }
$$

If $\Phi^{2}=1$ and $\mu_{\Phi i}=\mu_{i}$, then

$$
\left\langle f_{1} f_{2}\right\rangle_{\Lambda} \geqq\left|\left\langle f_{1} \phi f_{2}\right\rangle_{\Lambda}\right| \text {. }
$$

Proof. This follows at once from Equation (2.5) for $n=2$.

4 Cf., e.g., [4], Equations VIII, 23/24 
Connection with Griffith's Inequalities. As a special case of the Main Lemma consider a $\Lambda$ and $\Phi$ with $\Lambda_{0} \equiv \phi$ so that $\Lambda=\Lambda_{+} \cup \Phi \Lambda_{+}$, and let $\mu_{i}=\mu_{\Phi i}$,

$$
J_{i \Phi j}=0 \quad \text { for } \quad i, j \in \Lambda_{+} .
$$

Then (iii) becomes $J_{i j} \leqq 0$ for $i \neq j \in \Lambda_{+}$(ferromagnetic on $\Lambda_{+}$), and $d \mu_{\Lambda}=d \mu_{\Lambda_{+}} d \mu_{\Phi \Lambda_{+}}$. Hence, in this case

$$
\int f_{1} \phi f_{2} d \mu_{\Lambda}=\left(\int f_{1} d \mu_{\Lambda_{+}}\right)\left(\int f_{2} d \mu_{\Lambda_{+}}\right)
$$

and Equation (2.8) leads to the usual GKS inequalities with respect to $\mu_{\Lambda_{+}}$[4].

\section{Applications to Ising Ferromagnets}

Common symmetries in Ising ferromagnets are translations, rotations, and reflections. We first discuss the latter.

We consider a hyperplane which is orthogonal to the unit vector $\beta$ and which passes through the point $\alpha \in \mathbb{R}^{d}$. By $\Theta_{\beta}^{\alpha}$ we denote the reflection with respect to this hyperplane. Then

$$
\Theta_{\beta}^{\alpha} j=j-2[(j-\alpha) \cdot \beta] \beta,
$$

and $\mathbb{Z}^{d}$ is mapped onto itself if and only if one of the following two conditions holds

a) $\beta= \pm(0, \ldots, 1,0, \ldots), \quad 2 \alpha \cdot \beta \in \mathbb{Z}$

b) $\beta=\frac{1}{\sqrt{2}}(0, \ldots, \pm 1,0, \ldots, \pm 1,0, \ldots), \quad \sqrt{2} \alpha \cdot \beta \in \mathbb{Z}$

Lemma 3.1. Let the matrix $J=\left\{J_{i j} ; i, j \in \mathbb{Z}^{d}\right\}$ be translation invariant, $J_{i j}=J_{j-i}$. If $J$ is invariant under $\Theta_{\beta}^{0}$ then so it is under $\Theta_{\beta}^{\alpha}$ for any $\alpha$ with $2 \alpha \in \mathbb{Z}^{d}$.

Proof. By Equation (3.1), the $\alpha$-dependent terms cancel. QED.

Now consider an even Ising ferromagnet on $\mathbb{Z}^{d}$ with constant external field, whose interaction is $\Theta_{\beta}^{\alpha}$-invariant for some $\beta$. Consider a finite $\Theta_{\beta}^{\alpha}$-invariant sublattice $\Lambda$ of $\mathbb{Z}^{d}$ and impose free or periodic boundary conditions ${ }^{5}$. For the former we define $\Lambda_{0}$ simply as the plane of reflection intersected with $\Lambda$ (which may be empty), in case of periodic boundary conditions $\Lambda_{0}$ shall also contain the points which are identified. As $\Lambda_{+}$we choose the intersection of $\Lambda$ with one of the half spaces determined by the plane (minus $\Lambda_{0}$ ), in the positive direction of $\beta$, say. If in addition

$$
\left|J_{i \Theta_{\beta}^{\alpha} j}\right| \leqq\left|J_{i j}\right| \quad \text { for } \quad i \neq j, \quad i, j \in \Lambda_{+}
$$

then the assumptions of the Main Lemma are satisfied.

Let $H_{\alpha \beta}^{+}$denote the half space

$$
H_{\alpha \beta}^{+}=\left\{j \in \mathbb{Z}^{d} ;(j-\alpha) \cdot \beta \geqq 0\right\},
$$

5 One can also consider other reflection invariant boundary conditions 
let $\vartheta_{\beta}^{\alpha}$ implement $\Theta_{\beta}^{\alpha}$,

$$
\vartheta_{\beta}^{\alpha} \sigma_{j}=\sigma_{\Theta_{\beta}^{\alpha} j}
$$

and let, for a set $B \subset \mathbb{Z}^{d}, C_{+}(B)$ denote the set of all polynomials in $\left\{\sigma_{j} ; j \in B\right\}$ with positive coefficients ${ }^{6}$. Letting $\Lambda \rightarrow \mathbb{Z}^{d}$ (always taking $\Theta_{\beta}^{\alpha}$-invariant $\Lambda$ 's and the above boundary conditions) one obtains in the thermodynamical limit, from the Main Lemma and Corollary 2.2,

$$
\begin{aligned}
& \left\langle\prod\left(f_{k} \pm \vartheta_{\beta}^{\alpha} f_{k}\right)\right\rangle \geqq 0, \\
& \left\langle f_{1} f_{2}\right\rangle \geqq\left|\left\langle f_{1} \vartheta_{\beta}^{\alpha} f_{2}\right\rangle\right|, \quad f_{k} \in C_{+}\left(H_{\alpha \beta}^{+}\right) .
\end{aligned}
$$

From this we are going to derive some more concrete relations which apply in particular to the n.n. Ising model.

Theorem 3.1. Consider an even Ising-ferromagnet with constant external field. Let the interaction satisfy

(i) $J_{i j}=J_{j-i}$ (translation invariance)

(ii) $J_{\left(i_{1}, i\right)}=J_{\left(-i_{1}, i\right)}$ (reflection invariance)

(iii) $J_{\left(i_{1}, i\right)}$ is monotone decreasing ${ }^{7}$ in $i_{1}$ on $\left\{i_{1} \geqq 1\right\}$, for fixed $\underline{i}$.

Let the thermodynamical limit be taken with free or periodic boundary conditions, and let $T_{\tau}$ denote translation by $\tau$ in 1-direction. Then, for $f \in C_{+}\left(\left\{j ; j_{1}=a\right\}\right)$, $g \in C_{+}\left(\left\{j ; j_{1} \geqq a\right\}, a \in \mathbb{Z}\right.$, one has

$$
\left\langle f T_{\tau} g\right\rangle \geqq\left\langle f T_{\tau}, g\right\rangle \geqq 0 \quad \text { for } \quad 0 \leqq \tau \leqq \tau^{\prime} .
$$

In particular, $\left\langle\sigma_{0} \sigma_{\left(i_{1}, i\right)}\right\rangle$ is monotone decreasing in $i_{1}$ on $\left\{i_{1} \geqq 0\right\}$.

Proof. By (iii) the growth condition Equation (3.3) is satisfied for any half-space $H_{\alpha \beta}^{+}$ with $\beta=(1, \underline{0})$. By (ii) and Lemma $3.1, J$ is invariant under any $\Theta_{\beta}^{\alpha}$ with $\beta=(1, \underline{0})$ and any $\alpha$ with $2 \alpha \in \mathbb{Z}^{d}$. We take $\alpha=\left(a-1 / 2\left(\tau^{\prime}-\tau\right), \underline{0}\right)$ in Equation (3.7). Then $f, T_{\tau} g \in C_{+}\left(H_{\alpha \beta}^{+}\right)$and

$$
\vartheta_{\beta}^{\alpha} f=T_{-\left(\tau^{\prime}-\tau\right)} f .
$$

Equation (3.8) now follows from Equation (3.7) and translation invariance. QED.

Remark. For the n.n. Ising model Equation (3.8) has been proved in [1] and [2]. The growth condition is trivially satisfied for n.n. interactions. We note that for n.n.n. interactions and reflections at hyperplanes $\left\{j_{1}=n\right\}$ with integer $n$ the growth Condition (iii) of the Main Lemma is automatically fulfilled.

Monotonicity along diagonals is obtained by considering reflections at the "diagonal hyperplanes" $8\{i ; \cdot \beta=0\}$ where

$$
\beta=\frac{1}{\sqrt{2}}(0, \ldots, \pm 1,0, \ldots, \pm 1,0 \ldots) .
$$

\footnotetext{
6 Throughout one can also take $C_{+}(B)$ to denote the positive cone generated by the functions $h\left(x_{j}\right)$ in Lemma 2.1 with $j \in B$

7 Not necessarily strictly monotone

8 For the spin $1 / 2$ n.n. Ising reflections at diagonal hyperplanes were also considered in [2]
} 
Theorem 3.2. Consider an even Ising ferromagnet with constant external field. Let the interaction satisfy

(i) translation invariance,

(ii) invariance under reflection at the diagonal hyperplane $\{i ; i \cdot \beta\}=0$ with $\beta$ as in Equation (3.9),

(iii) $J_{i j}$ is monotone decreasing ${ }^{7}$ with the distance of $i$ and $j(i \neq j)$.

Let the thermodynamical limit be taken with free boundary conditions, and let $T_{i}$ denote translation by $i \in \mathbb{Z}^{d}$. Then, for any $\beta$ of Equation (3.9) and any

$$
\begin{aligned}
& f \in C_{+}(\{j ; j \cdot \beta=a / \sqrt{2}\}) \\
& g \in C_{+}(\{j ; j \cdot \beta \geqq a / \sqrt{2}\})
\end{aligned} \quad a \in \mathbb{Z}
$$

one has

$$
\left\langle f T_{\tau \sqrt{2} \beta} g\right\rangle \geqq\left\langle f T_{\tau^{\prime} \sqrt{2} \beta} g\right\rangle \geqq 0, \quad 0 \leqq \tau \leqq \tau^{\prime} ; \tau, \tau^{\prime} \in \mathbb{N} \cup\{0\} .
$$

In particular, if (ii) holds for each diagonal hyperplane then, for $i_{1}, \ldots, i_{d} \geqq 0$,

$$
\left\langle\sigma_{0} \sigma_{i}\right\rangle \geqq\left\langle\sigma_{0} \sigma_{\left(0, \ldots, i_{1}+\ldots+i_{n+1}, \ldots, i_{d}\right)}\right\rangle .
$$

If, in addition, invariance under each reflection $i \rightarrow\left(i, \ldots,-i_{k}, i_{k+1}, \ldots\right)$ holds then, for $i \in \mathbb{Z}^{d}$,

$$
\left\langle\sigma_{0} \sigma_{i}\right\rangle \geqq\left\langle\sigma_{0} \sigma_{\left(\Sigma\left|j_{v}\right|, \underline{Q}\right)}\right\rangle \text {. }
$$

Proof. By (iii) the growth condition Equation (3.3) is satisfied for any half-space $\mathrm{H}_{\alpha \beta}^{+}$ for $\beta$ as in Equation (3.9). By Lemma 3.1 and (ii) $J$ is invariant under any such $\Theta_{\beta}^{\alpha}$ when $2 \alpha \in \mathbb{Z}^{d}$. In Equation (3.7) we take $\alpha=\left(a-\tau^{\prime}+\tau\right) \beta / \sqrt{2}$. Then $f, T_{\tau \sqrt{2} \beta} g \in C_{+}\left(H_{\alpha \beta}^{+}\right)$ and, by Equation (3.1),

$$
\vartheta_{\beta}^{\alpha} f=T_{-\sqrt{2}\left(\tau^{\prime}-\tau\right) \beta} f .
$$

Equation (3.10) now follows from Equation (3.7) and translation invariance.

To prove Equation (3.11) we note that by reflection invariance we can always assume $i_{1} \leqq \ldots \leqq i_{n}$. Choosing $\beta$ and $\tau$ such that

$$
\begin{aligned}
\beta_{v} & =\frac{1}{\sqrt{2}}\left(-\delta_{1 v}+\delta_{n v}\right) \\
\tau & =i_{1}
\end{aligned}
$$

we get from Equation (3.10)

$$
\left\langle\sigma_{0} \sigma_{i}\right\rangle \geqq\left\langle\sigma_{0} \sigma_{\left(0, i_{2}, \ldots, i_{1}+i_{n}, i_{n+1}, \ldots\right)}\right\rangle \text {. }
$$

Equation (3.11) is now obtained inductively, and the rest is trivial. QED.

The result shows that for given $\sum\left|i_{v}\right|$ the two point function is minimal on the coordinate axes and maximal on the diagonals. The results hold in particular for the n.n. Ising model since assumptions (i)-(iii) of Theorem 3.2 are satisfied. In the 2dimensional n.n. Ising model it is known that, for $T>T_{C},\left\langle\sigma_{0} \sigma_{i}\right\rangle$ is not an (isotropic) function of the distance $r=\left(i_{1}^{2}+i_{2}^{2}\right)^{1 / 2}$, not even asymptotically [5]. 
In special cases other choices of $\Lambda_{+}$are possible. A trivial example for $d=2$ is $J_{i j}=-J$ for $|i-j|=2$ and zero otherwise. For a reflection at the line $j_{1}=-1 / 2$ one may take $\Lambda_{+}$to consist of every second line parallel to the $j_{2}$-axis. The growth condition is then satisfied. However, this example corresponds to two independent n.n. Ising models and leads to nothing new.

We now briefly discuss possible applications of the Main Lemma to other symmetry transformations.

Inversion $j \mapsto-j$. The set of invariant elements is $\Lambda_{0}=\{0\}$. For $\Lambda_{+}$one may take e.g.,

$$
\Lambda_{+}=\bigcup_{v=1}^{d}\left\{j \in \mathbb{Z}^{d} \cap \text { box } ; j_{1}=\ldots=j_{v-1}=0, j_{v}>0\right\} .
$$

Again it is difficult to satisfy the growth Condition (iii) of the Main Lemma, at least it is not fulfilled for n.n. and n.n.n. interactions.

Rotations. To be specific we consider $d=2$ and a rotation $\Phi$ by $\pi$ so that $\Phi^{2}=1$. Again $\Lambda_{0}=\{0\}$, and as $\Lambda_{+}$one may take, e.g.,

$$
\Lambda_{+}=\left\{j \in \mathbb{Z}^{2} \cap \text { box } ; j_{1}>0 \text { or } j_{1}=0, j_{2}>0\right\} \text {. }
$$

Again the growth condition is not satisfied for n.n. and n.n.n. interactions.

Translations (with periodic boundary conditions). In this case $\Phi^{2} \neq 1$ so that Condition (iv) of the Main Lemma is not automatically satisfied. Periodicity of the interaction will assure it, e.g. for $d=1$, if $J_{0 k}=J_{0 k+2}$ for $k \neq 0$. Also the growth condition can be satisfied for suitable $J_{0 k}$, but such a model seems not very interesting.

Triangular and Other Lattices. The Main Lemma can also be applied to the triangular lattice with reflection invariant n.n. interaction. The resulting inequalities are similar to those of Theorems 3.1 and 3.2. It may be that rotations and inversions could be usefully exploited in more general lattices, also nonlinear transformations might in some cases be considered.

Acknowledgments. I would like to thank K. Baumann, H. J. Borchers, A. Messager, S. Miracle-Sole, H. Roos, R. Schrader, and J. Yngvason for stimulating discussions.

\section{References}

1. Schrader, R.: New correlation inequalities for the Ising model and $P(\phi)$ theories. Phys. Rev. B 15, 2798 (1977)

2. Messager,A., Miracle-Sol,S.: Correlation functions and boundary conditions in the Ising ferromagnet. Preprint, Marseille 1977

3. Ginibre,J.: General formulation of Griffith's inequalities. Commun. math. Phys. 16, 310 (1970)

4. Simon, B.: The $P(\phi)_{2}$ Euclidean (quantum) field theory. Princeton: Princeton University Press 1974

5. McCoy,B.M., Wu,T.T.: The two-dimensional Ising model. Cambridge: Harvard University Press 1973

Communicated by E. Lieb 